\title{
Cambios en los patrones de consumo entre los estudiantes de periodismo: de prosumidores mediáticos a profesionales de la información
}

\author{
Juan Carlos SuÁrez Villegas \\ jcsuarez@us.es \\ Universidad de Sevilla \\ Jesús CRuz Álvarez \\ jcruz12@us.es \\ Universidad de Sevilla
}

Recibido: 6 de febrero de 2014

Aceptado: 8 de septiembre de 2014

\section{Resumen}

El presente estudio analiza los cambios de consumo informativo desarrollados con el advenimiento del entorno digital de la comunicación y las nuevas relaciones entabladas entre consumidores, contenidos y productores de información. Para ello, se ha llevado a cabo un cuestionario semiestructurado entre 140 alumnos y alumnas de último curso de periodismo de la Universidad de Sevilla, concretamente en el marco de la asignatura de Ética y Deontología Periodística. A partir de las siete preguntas planteadas, se ha perseguido conocer los hábitos y opiniones del alumnado en torno a cuestiones como el uso de redes sociales, preferencias mediáticas, o identificación de los principales dilemas a los que se enfrenta la profesión.

Palabras clave: Comunicación Digital, Audiencias, Consumo de Medios, Ética de la Comunicación, Redes Sociales, Periodismo Participativo, Estudiantes.

\section{Changes in consumption patterns among students of journalism: from media prosumers to journalists}

\begin{abstract}
This study analyzes the changes in news consumption developed with the advent of digital communication environment and new relations established between consumers, producers and contents. To do this, we have conducted a semi-structured questionnaire among 140 students of final year of journalism at the University of Seville, specifically in the context of the subject of Journalistic Ethics. From the seven questions, was persecuted to know the habits and opinions of students on issues such as the use of social networking, media preferences, or identification of the major dilemmas facing the profession.

Keywords: Digital Communication, Audiences-Media, Consumption-Media, Ethics-Social Networks, Participatory Journalism, Students.

\section{Referencia normalizada}

SUÁREZ VILLEGAS, Juan Carlos y CRUZ ÁLVAREZ, Jesús (2015): “Cambios en los patrones de consumo entre los estudiantes de periodismo: de prosumidores mediáticos a profesionales de la información”. Estudios sobre el Mensaje Periodístico. Vol. 21, Núm. 1 (enero-junio), págs.: 615-628. Madrid, Servicio de Publicaciones de la Universidad Complutense.
\end{abstract}

Sumario: 1. Introducción. 2. Material y métodos. 3. Análisis y Resultados. 4. Discusión y Conclusiones. 5. Referencias bibliográficas. 


\section{Introducción}

Los procesos de convergencia desarrollados de forma simultánea a nivel tecnológico, económico y organizacional en el seno de los grandes grupos de comunicación de todo el mundo, ha incidido en la asunción de un nuevo tipo de periodismo multimodal, multiplataforma y polivalente que ha modificado sustancialmente las prácticas de los profesionales en las redacciones y en las relaciones entabladas con el entorno. La información ha dejado de ser un producto inequívoco y acabado sujeto a la jerarquía de la cultura editorial de un medio concreto. La confluencia de las nuevas tecnologías de la información y la concentración empresarial de los medios, unido a su vez con la adopción por parte de los periodistas de roles desempeñados hasta ahora por otros actores, ha emplazado a la profesión periodística a un escenario marcado por la incertidumbre en el que no existen guías incuestionables para un futuro que muta constantemente.

Nunca antes en la historia se había consumido tanta información, sin embargo, la credibilidad y autoridad moral mantenida tradicionalmente por los periodistas y, por ende, por los medios de comunicación, se han visto reducidas de forma evidente en los últimos años. Un claro ejemplo de ello es el último barómetro del Centro de Investigaciones Sociológicas (CIS, 2013) de España en torno a la confianza generada por las distintas profesiones, en el que los periodistas aparecían distinguidos como los menos valorados por la sociedad, tan sólo superados en descrédito por el colectivo de jueces.

Paralelamente al derrumbamiento progresivo de la prensa tradicional, manifestado por la pérdida inexorable de lectores, se ha producido una migración masiva a los medios digitales que ha redundado en la acumulación de audiencias millonarias. No obstante, este aparente éxito no se ha traducido en el desarrollo cualitativo de una profesión precarizada y con altos niveles de desempleo. Más bien ha supuesto el surgimiento de dilemas éticos vinculados con la presión a la insta una rutina informativa basada en la actualización permanente, tales como la dificultad para verificar de forma independiente los hechos narrados o la confusión generada por la incorporación de nuevos actores al proceso informativo.

Los periodistas han perdido el monopolio de la verdad informativa a raíz de la difuminación de las fronteras entre los productores de la información y "la gente antes conocida como audiencias" (Rosen, 2006). Es lo que Deuze (2008) ha denominado como "periodismo líquido", en alusión al trabajo del sociólogo polaco Zygmunt Bauman, en la medida en que todo lo relacionado con la profesión adquiere un carácter provisional, inestable e hipertextual, fruto de la adopción de una actitud más activa de la ciudadanía a partir del uso de las nuevas herramientas digitales. El académico español Manuel Castells definió este fenómeno como la "autocomunicación de masas" (2009), o lo que es lo mismo, la individualización del consumo y la producción de información de acuerdo a una necesidad del lector a filtrarse en el proceso informativo, ya sea comentando una noticia, enviando material a los medios o retweeteando un enlace.

A este respecto, Alfred Hermida ha acuñado el término de periodismo ambiental para referirse al sistema mediante el cual se ofrece al usuario la posibilidad de recopilar, comunicar, compartir y consumir información de forma constante y con distin- 
tos propósitos de acuerdo a un estado de conciencia siempre alerta al menor estímulo del entorno (2010). Quizás la catalogación más paradigmática de este nuevo usuario híbrido sea la aportada hacía varias décadas por el tecnólogo Alvin Toffler (1971), quien comenzó a utilizar la palabra prosumer (prosumidor) para referirse a un individuo que no sólo se contentaba con recibir los contenidos de la industria cultural, sino de se disponía a buscarlos y demandarlos. Más reciente es el término produser, acuñado por Axel Bruns (2005) para definir una práctica de producción informativa más abierta y colaborativa que entronca en cierto modo con el denominado periodismo ciudadano, descrito por Bowman y Willis como el acto por el cual un ciudadano o grupo de ellos juegan un rol activo en el proceso de recolección, análisis, edición o difusión de noticias e información (2003).

Los usuarios digitales demandan así una participación activa en el proceso informativo que trasciende la mera publicación de comentarios y que persigue su introducción en las distintas etapas de producción de la noticia como un elemento más con capacidad de influencia en el resultado final. Ante este nuevo escenario, los medios de comunicación convencionales, temerosos de perder aún más la hegemonía en la producción y difusión de contenidos informativos, han aplicado estrategias híbridas, en muchos casos siguiendo la metodología del ensayo y error, tendentes a crear en torno a ellos una comunidad de usuarios ajustados a unos cauces de participación que reservan a los profesionales una serie de tareas consideradas puramente periodísticas, como son el filtrado y la edición de la información. Es decir, si bien es cierto que ya no es posible hacer periodismo al margen de las audiencias, los periodistas siguen esforzándose en mantener a estas en su lugar, estableciendo una marcada diferencia entre la comunidad y la redacción, tal y como se ha desvelado en numerosas investigaciones (Domingo et al. 2008; Harrison 2009; Singer et al. 2011).

No obstante, al igual que la información ya no es un producto exclusivo de los medios de comunicación, la capacidad de los usuarios para comentar, compartir y consumir de forma transversal contenidos va más allá de las posibilidades de participación ofrecidas por la comunidad alojada en los medios. De hecho, la arquitectura primordial del periodismo ambiental construido entre periodistas y audiencias (Burns, 2010) se basa principalmente en el rápido desarrollo de redes sociales como Facebook y Twitter, que no sólo han contribuido a reforzar el papel del usuario digital de información en su vertiente más interactiva, sino que han modificado sustancialmente las rutas de acceso y consumo de contenidos periodísticos alterando de este modo los hábitos de los lectores y las relaciones mantenidas con los medios de comunicación. No en vano, las redes sociales ya son la segunda fuente de importancia de generación de tráfico online sólo por detrás de Google (Olmstead et al., 2011), y la tendencia es que sigan incrementando su influencia entre las audiencias con base a su fácil accesibilidad (más aún con la rápida extensión de los dispositivos móviles), la inmediatez de la información o las posibilidades de interacción y recomendación entre usuarios.

Las empresas de comunicación no han permanecido ajenas a este fenómeno y están utilizando las redes sociales como herramientas de generación de tráfico hacia sus webs, sin embargo han obviado en gran medida su potencial en la construcción de comunidades virtuales y en la creación de vínculos de compromiso con sus audiencias 
(Messner, Linke and Esford, 2011:20). La mayoría de las cuentas oficiales de los medios únicamente publican contenidos autopromocionales, y su interacción con los usuarios es casi inexistente. La tradicional lealtad a la cabecera de los lectores, manifestada en la compra diaria de un periódico determinado, se ha diluido entre la amalgama de medios, periodísticos o no, que cruzan la Red y con los que se guardan escasos lazos emocionales. Los medios y los periodistas, "a pesar de que aún detentan cierto poder en el control de los flujos informativos, han pasado a ser otra voz entre muchas en la comunicación pública" (Deuze, 2008:12). Ahí radica el núcleo de un cambio que afecta a los patrones de consumo, a la identidad del periodista y a la propia información.

Y en ese marco donde se inserta la investigación aquí planteada. En una coyuntura de incuestionable incertidumbre no sólo en relación a los dilemas suscitados por el nuevo entorno digital sino incluso a la supervivencia del periodismo como profesión independiente, se antoja fundamental analizar los cambios producidos en el consumo social de la información para, desde este punto, descifrar las tendencias de cuyo desarrollo depende en gran parte el futuro de la comunicación pública. A este respecto, la investigación en esta materia debe prestar una especial atención al nuevo papel adoptado por las audiencias activas o prosumidores de información, analizando en primer término si efectivamente estas están caracterizadas por un dinamismo generalizado en cuanto a participantes habituales tanto en medios como en otros canales sociales. Para ello, en el presente estudio hemos focalizado nuestra atención en las actitudes mantenidas por estudiantes universitarios de último curso de la licenciatura en Periodismo, en relación a sus patrones de consumo informativo, su actividad en redes sociales o su opinión sobre las nuevas vías de participación ciudadana en el entorno comunicativo digital. Dada su condición dual de ciudadanos jóvenes y, por tanto, en contacto con las nuevas tecnologías, formatos y soportes, así como futuros periodistas, consideramos de indudable interés su aportación al objeto de estudio planteado.

\section{Material y métodos}

La presente investigación se basa en el análisis cualitativo de una encuesta semiestructurada de siete preguntas realizada a 140 alumnos y alumnas de quinto curso de la licenciatura de Periodismo de la Universidad de Sevilla. La encuesta ha sido desarrollada en el marco de la asignatura de Ética y Deontología Periodística, impartida por el profesor Juan Carlos Suárez Villegas. La mayoría de las preguntas ofrece la posibilidad de dar respuestas abiertas y razonadas, lo que nos ha permitido conocer en mayor profundidad las opiniones y comportamientos habituales de los alumnos en su relación con los medios de comunicación y las redes sociales. A continuación, analizaremos la pertinencias de las distintas preguntas planteadas en la encuesta.

- Pregunta 1: ¿Qué tipo de medios de comunicación (digitales o analógicos) utilizas en mayor medida para informarte? Comenta las razones de esta preferencia.

Mediante esta pregunta se pretende conocer los hábitos informativos del alumnado universitario de periodismo para calibrar de este modo la hipotética migración de los medios de comunicación analógicos (prensa escrita, radio, revistas, etc.) hacia los nuevos formatos digitales, fundamentalmente por parte de los jóvenes. De igual forma, 
se solicitan las razones por las que se decantan por uno u otro formato, permitiéndonos así glosar las características positivas que invitan a su respectivo uso.

- Pregunta 2: ¿Crees que la información suministrada por los medios de comunicación digitales es sustancialmente diferente a la de los tradicionales? ¿en qué sentido? Razona tu respuesta.

Con esta pregunta se busca conocer las diferencias o similitudes que, de acuerdo con los alumnos, existen entre sendos formatos. La formación periodística del alumnado contribuye asimismo a ofrecer una visión más completa y razonada de esta comparación, ya que se presupone su conocimiento de ambos soportes. Del mismo modo, los resultados de esta pregunta pueden ser cruzados con los de la anterior y averiguar si las preferencias manifestadas previamente guardan relación con las características atribuidas en esta.

- Pregunta 3: ¿Tienes algún medio de comunicación predilecto para informarte o acudes a varios de diferentes tendencias? Indica las razones.

Se trata de una pregunta más concreta que pretende analizar el grado de pluralidad informativa consultada por el alumnado y su vínculo con el método de acceso a los contenidos. Es decir, si bien el lector tradicional de prensa compraba generalmente un solo periódico, el lector digital se caracteriza más por acudir a varias cabeceras, en gran medida gracias a redes sociales como Twitter, en la que se suelen seguir a numerosos medios de comunicación. Al fin y al cabo, el acceso es fácil y gratuito. De ser corroborado, se trata de un cambio de tendencia en el consumo informativo transcendental, al posibilitar la conformación de una opinión en en lector más amplia y heterogénea.

- Pregunta 4: ¿Utilizas las redes sociales para obtener información? ¿Con qué frecuencia? ¿Accedes desde las redes sociales a la información o simplemente lees los titulares en tu muro?

Con estas preguntas nos introducimos definitivamente en el entorno digital y, en concreto, en el uso de las redes sociales por parte de los encuestados. Se trata de conocer si estas redes son utilizadas con afán informativo, la periodicidad de la consulta y el modo en el que es consumida. Las características del denominado periodismo ambiental nos arroja a un escenario donde los lectores acuden a los medios, de forma repetida a lo largo del día en busca de actualizaciones y noticias de última hora, sin embargo, también contribuye a una lectura más superficial de los contenidos, en muchas ocasiones limitada a los titulares. Del mismo modo, se intenta averiguar la conducta de los encuestados en el acceso a esos contenidos, es decir, si acuden directamente a los medios o si son redireccionados a partir de Facebook o Twitter.

- Pregunta 5: ¿Sueles compartir información a través de tu perfil en redes sociales? Si es así, ¿qué tipo de información compartes y con qué objetivo?

Íntimamente relacionada con la pregunta anterior, ya que en cierto modo es una consecuencia de la misma, se da un paso más en el proceso del consumo social de la información, centrándose en la etapa de recomendación de contenidos. Tal y como veíamos en la introducción, las redes sociales son ya la segunda fuente de generación de tráfico online gracias a los enlaces tanto de las cuentas oficiales de los medios como de los compartidos por 'amigos' y 'seguidores'. En esta segunda tendencia es en la que 
nos centramos para confirmar la preeminencia de la denominada serendipia informativa, es decir, cuando accedes a la información sin buscarla de forma autónoma, sino a partir de las recomendaciones de los contactos. También pretendemos conocer las razones de esta práctica de compartir información.

- Pregunta 6: Como ciudadano/a, ¿crees que la participación de los usuarios en los medios digitales contribuye de forma positiva al consumo social y democrático de información? ¿Piensas lo mismo como futuro/a periodista? Razona tu respuesta.

Con esta pregunta nos valemos de la dualidad de los encuestados como ciudadanos y futuros periodistas para conocer su opinión sobre la participación de los usuarios en la Red. Se trata de una posibilidad idónea para la investigación, ya que muchos de los encuestados han venido desarrollando en los últimos años proyectos y colaboraciones en medios digitales periodísticos o no en calidad de ciudadanos, condición que están próximos a sustituir (profesionalmente hablando) por la de periodistas. Es curioso hacer notar el desdoblamiento de esta naturaleza en las respuestas dadas, lo cual nos ofrece un botón de muestra de la complejidad del asunto.

- Pregunta 7: Explica desde tu punto de vista como ciudadano y futuro periodista cuáles son los principales dilemas éticos que se plantean en el nuevo entorno comunicativo digital.

Con esta última pregunta volvemos a utilizar la condición híbrida de los encuestados para, en esta ocasión, analizar los principales dilemas éticos que, desde su opinión, plantea el entorno digital. El objetivo es confrontar los problemas éticos relacionados con la profesión con aquellos otros sobrevenidos desde el exterior, como puede ser la emergencia del periodismo ciudadano.

\section{Análisis y Resultados}

Dado el carácter cualitativo perseguido en la confección del cuestionario, las respuestas aportadas por los encuestados varían en cierto modo de acuerdo a las diferentes visiones de estos sobre los asuntos tratados, generando un valor añadido al estudio a partir de los razonamientos esgrimidos libremente por los alumnos. A pesar de ello, y con objeto de obtener algunos resultados cuantificables, hemos elaborado gráficas que agrupan las principales tendencias observadas en las distintas preguntas. A continuación, analizaremos ordenadamente los datos recabados.

\section{Pregunta 1}

La mayoría de los encuestados (122 sobre 18) asegura utilizar de forma generalizada los medios de comunicación digitales para informarse diariamente. Por el contrario, la preeminencia de la prensa escrita entre el alumnado es residual, quedando circunscrita a un número reducido de encuestados que continúan comprando periódicos en el kiosco. De hecho, dentro de este pequeño grupo, el consumo de prensa digital sigue siendo habitual, aunque se complemente con la costumbre de leer en papel, fundamentalmente los fines de semana.

Lo más interesante de las respuestas obtenidas a esta primera pregunta no se halla en la confirmación, más o menos vislumbrada, del cambio patente de los hábitos de consumo entre la juventud universitaria, sino en las razones dadas para justificar esta 
migración hacia los medios digitales. Entre los beneficios más citados de los alumnos destaca la gratuidad extendida en el entorno digital, que se relaciona a su vez con el fácil acceso, la comodidad y la rapidez de consulta de los contenidos de diferentes medios. Esta condición permite la opción de comparar los enfoques y noticias de las cabeceras, redundando en un pluralismo informativo relacionado con la visión crítica de los encuestados con la línea editorial de los medios, tal y como veremos en la pregunta número 3.

Otra de las ventajas apuntadas es la actualización constante a la que está sujeta la información en los medios digitales, con una especial atención a noticias de última hora. Según los encuestados, esto da lugar a una información más concisa y versátil que se ajusta a sus necesidades como consumidores y al escaso tiempo con el que cuentan para desarrollar una actividades cada vez más efímera y compulsiva. Por el contrario, también se hace referencia a las posibilidades de contraste de la información que los medios digitales ofrecen a partir del carácter hipervincular de las noticias, permitiendo acudir a otros websites desde el contenido matriz.

En último término, la interactividad también ocupa un lugar destacado entre las razones dadas para legitimar la preferencia de los medios digitales, ya que permite un contacto más directo con la información, que además puede ser compartida. De acuerdo con algunos de los encuestados, la interactividad desvela el interés de los medios en la opinión de los lectores, en contraposición con el carácter más estático de los medios tradicionales.

Por su parte, aquellos que se decantan por la prensa escrita aseguran que la eligen por costumbre, por una visión bohemia del periodismo o por los suplementos agregados en las ediciones de los fines de semanas. De igual modo, reconocen que el consumo de esta prensa se ajusta a los momentos en los que tienen tiempo para leer con tranquilidad.

Además de la dicotomía entre prensa digital y en papel, varios encuestados consumen medios de comunicación analógicos como la radio y la televisión, principalmente por costumbre, por su fácil acceso y por la posibilidad de hacer otras tareas simultáneamente.

\section{Pregunta 2}

Una vez corroborada la hipótesis de la migración de la prensa tradicional al entorno digital por parte del sector poblacional más joven, así como las razones que la justifican, es preciso conocer si los encuestados creen que existen diferencias sustanciales entre sendos formatos. Si bien es cierto que la mayoría apunta que las diferencias son manifiestas (99 de los 140), cabe destacar un porcentaje relevante (25\%) que asegura que estas disparidades no son sustanciales y, por ende, no alteran de forma significativa los contenidos informativos.

En primera instancia, si analizamos al primer grupo, podemos desglosar a su vez otros dos subgrupos de acuerdo con las razones aportadas para justificar esas diferencias. Mayoritariamente, los encuestados argumentan los beneficios de la prensa digital (muchos de estos referidos en la pregunta anterior) como rasgos distintivos entre ambos formatos. Entre ellos, la inmediatez de las informaciones en la Red, la 
interactividad, el carácter multimedia de los contenidos, las posibilidades de profundizar gracias a los vínculos y las bases de datos, la pluralidad temática o el atractivo del diseño. Por el contrario, un número más reducido de encuestados afirman que el periódico tradicional es más fiable, de mayor calidad y la información es más extensa y exhaustiva, siendo de este modo la que aporta reflexión.

En el segundo grupo, es decir, aquellos que consideran que no existen grandes diferencias entre sendos formatos, únicamente reconocen de que existe un cambio de estilo, en la medida en que el periodismo digital es más visual y en el convergen diferentes herramientas. También se diferencia entre los medios nativos digitales y los tradicionales con versión on-line, ya que, si bien estos reproducen una información similar a sus matrices impresas, los nativos sí que ofrecen enfoques alternativos.

\section{Pregunta 3}

De acuerdo a los datos recogidos en la gráfica, la mayoría de los encuestados asegura acudir a varios medios de comunicación con distintas líneas editoriales para informarse. Otro porcentaje importante reconoce que suele consultar los más conocidos, mientras que una minoría admite que se informa a través de aquellos que considera cercanos a su ideología, o que sólo utiliza Twitter como medio de comunicación.

Los encuestados del primer grupo esgrimen que no confían en un medio de comunicación en concreto y aseguran que ninguno está en posesión de la verdad informativa. De hecho, algunos creen que informarse por distintas vías es un acto de responsabilidad. Por su parte, los que únicamente acuden a los tradicionales, fundamentalmente El País o El Mundo, apuntan que lo hacen por costumbre y por falta de Pregunta 3: ¿Tienes algún medio de comunicación predilecto o acudes a varios de diferentes tendencias? tiempo. En el caso de que sea una noticia de gran impacto o de especial interés, sí que intentan consultar otros de tendencias opuestas.

\section{Pregunta 4}

La mayoría de los encuestados reconoce que utilizan de forma habitual las redes sociales con fines informativos, mientras que un porcentaje menor afirma que las utilizan pero no como medio de comunicación. Tan sólo un grupo residual de alumnos asegura que no utilizan las redes sociales para ninguna tarea.

La dinámica de uso compartida por los encuestados del primer grupo es muy similar: entran asiduamente a sus redes sociales (en especial Twitter y Facebook), in- 
cluso varias veces al día, leen los titulares o tweets de las personas a las que siguen o con las que comparten amistad, y acceden a los contenidos si el titular o la materia les resulta interesante. $\mathrm{Mu}-$ chos reconocen que acceden desde el teléfono móvil, y a la hora de ampliar la información, utilizan el portátil o PC. Las

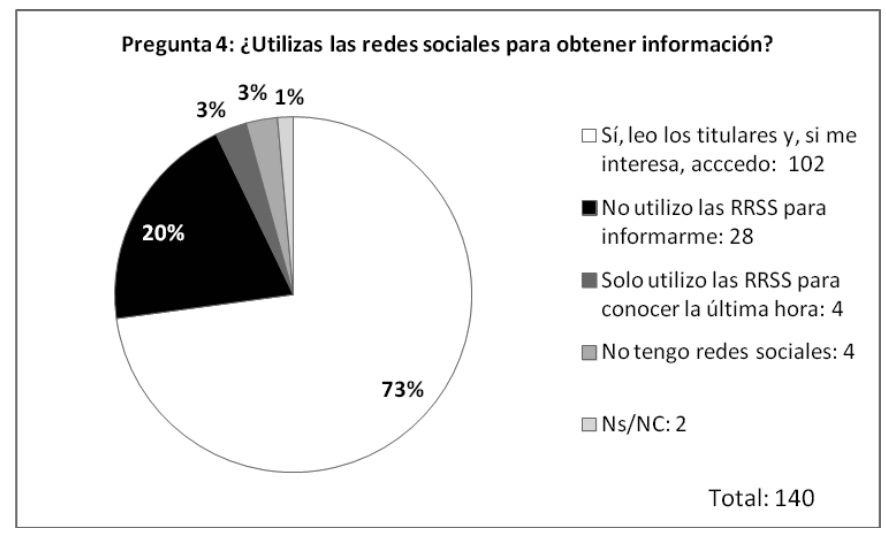
redes sociales están muy apegadas a la información de última hora, así como a las tendencias del momento. Algunos de los encuestados admiten que las redes sociales son herramientas fundamentales para estar al día de la actualidad del país y conocer los intereses de la sociedad.

Por su parte, los encuestados que no utilizan las redes sociales para informarse alertan de la proliferación de rumores y noticias sin contrastar que viajan por la Red, de ahí que no le confieran credibilidad a estas herramientas y prefieran acudir directamente a los medios de comunicación.

\section{Pregunta 5}

Según las respuestas recabadas, una mayoría de los alumnos comparte información a través de redes sociales, aunque cabe destacar que este porcentaje es menor al que figuraba en la pregunta anterior correspondiente a aquellos que consumían asiduamente información a través de estos canales. Una parte del alumnado afirma que nunca comparte información, mientas que un porcentaje mayor admite que lo hace sólo con

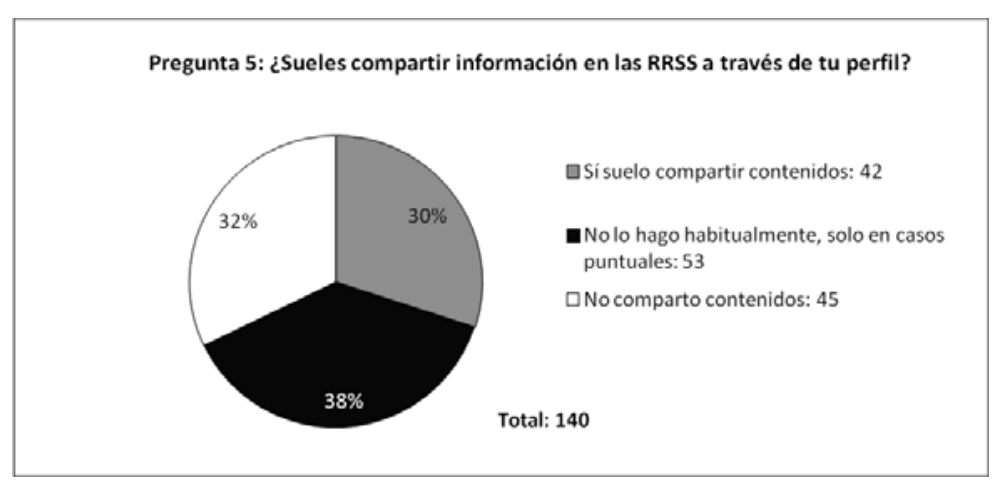

cuestiones de gran trascendencia social o personal.

Una parte de aquellos más activos en la tarea de compartir, lo hacen con el objetivo de concienciar a su entorno social o denunciar

situaciones que consideran injustas. La mayoría de ellos reconocen que el propósito de compartir información es generar debate, socializarse con el resto de amigos y seguidores, y hacer llegar información trascendente a aquellos que no suelen acudir a medios de comunicación. 


\section{Pregunta 6}

Dada la naturaleza dual de la pregunta, los resultados obtenidos ofrecen perspectivas heterogéneas del asunto. A pesar de que la defensa de la participación mediática de la ciudadanía como estímulo para el desarrollo democrático del país es la opción mayoritaria para el alumnado en periodismo, los matices aportados a esta opinión añaden complejidad a la visión global del análisis. De este modo, dentro del grupo de los que están a favor de la participación, hay varios encuestados que puntualizan que esta

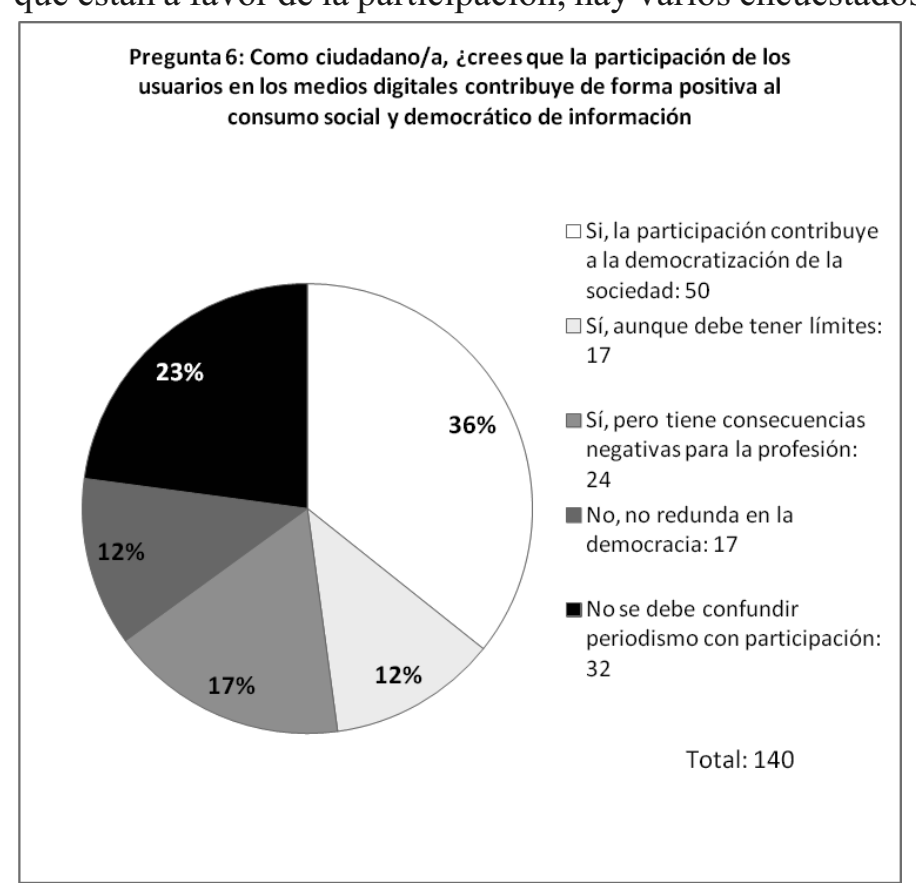

participación debe tener ciertos límites y debe ser ejercida con responsabilidad. También algunos reconocen que, si bien es cierto que esta favorece la democratización de la sociedad, tiene consecuencias negativas para la profesión. Por su parte, otro porcentaje está de acuerdo con una participación al nivel de difusión e interpretación de la información, pero no en la fase de producción, coto que se reserva para los profesionales.

En la posición contraria, aunque en menor número, existen opiniones de que la participación ciudadana no contribuye al diálogo, que está mediatizada o que, sencillamente, no existe. En una postura similar, algunos encuestados valoran que estas manifestaciones de los usuarios no pueden ser consideradas como periodismo, estableciendo así una diferencia manifiesta entre el profesional, que conoce las técnicas y los conocimientos teóricos indispensables, y el ciudadano. La postura generalizada es que la participación de los prosumidores mediáticos es un arma de doble filo que termina por afectar al oficio, aunque pueda ser beneficiosa para la sociedad.

\section{Pregunta 7}

Con las respuestas recogidas en esta pregunta se pueden glosar los principales problemas que, de acuerdo con los futuros periodistas, acucian a la profesión. Entre los más destacados aparece la inmediatez y la falta de tiempo a la hora de confeccionar la información, elemento que se relaciona a su vez con la falta de veracidad presente en las noticias. En segundo lugar, el intrusismo laboral y el periodismo ciudadano se antojan como importantes dilemas en el desarrollo de un periodismo ético, entre otras 
razones porque la carencia de formación y conocimientos de las normas deontológicas redunda en la elaboración de una información atenta a estos códigos. Por otra parte, el sensacionalismo es una preocupación importante

Pregunta 7: Explica desde tu punto de vista como ciudadano y futuro periodista cuáles son los principales dilemas éticos que se plantean en el nuevo entorno comunicativo digital

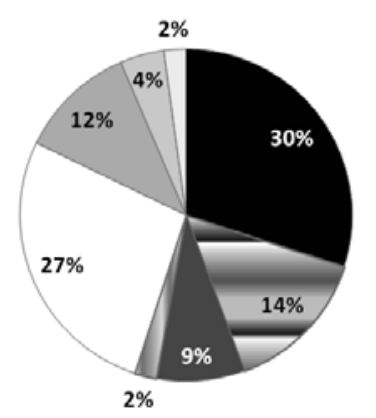

- Falta de tiempo, inmediatez: 42

घSensacionalismo: 20

m Gratuidad: 12

口Privacidad: 3

$\square$ Intrusismo/periodismociudadano: 38

a Falta de filtros de control: 16

$\square$ Precariedad laboral: 6

$\square$ Saturación informativa: 3

Total: 140

para una buena parte de los encuestados y, en concreto, la ponderación entre hechos noticiosos de interés y contenidos morbosos.

Con menor representación entre las respuestas del alumnado, la falta de ética, la saturación informativa, la gratuidad de los contenidos, las rutinas productivas, la falta de privacidad o la precariedad laboral son otros problemas derivados del entorno digital. Es curioso, de igual modo, hacer notar la inquietud de algunos encuestados por la falta de filtros y organismos de control en los flujos informativos digitales, apostando por una suerte de censura de carácter ético que impida la difusión de videos, fotografías o textos que atenten contra los derechos fundamentales de las personas.

\section{Discusión y Conclusiones}

La encuesta realizada a 140 alumnos y alumnas de último curso de la licenciatura de Periodismo de la Universidad de Sevilla nos ha permitido analizar algunas de las tendencias planteadas por el cambio de escenario en el consumo de la comunicación social, con el paso de una audiencia marcada por cierta pasividad a las denominadas audiencias activas o prosumidores mediáticos. Si bien el presente estudio recoge una muestra humilde para extraer conclusiones generales sobre un asunto de suma complejidad, la naturaleza híbrida de los encuestados, ciudadanos jóvenes e inminentes periodistas al mismo tiempo, confieren un valor añadido a la investigación que nos permite ahondar más en algunas de las cuestiones desde una doble perspectiva.

Entre las primeras certezas analizadas destaca el consumo de información en formato digital, la opción mayoritariamente elegida por el alumnado en detrimento de la prensa tradicional, cuya pérdida de lectores, más aún si cabe entre el sector joven, continúa su marcha inexorable. La extensión de los dispositivos móviles también ha contribuido a la rutinización del consumo digital de información, en la medida en que ya ni siquiera es necesario contar con un ordenador (PC o portátil) con conexión a Internet para acceder a los contenidos, sino que desde el smartphone se pueden consultar las últimas noticias, ya sea desde la aplicaciones de los medios, desde el navegador o desde las redes sociales. De hecho, según datos de Comscore (2012), la penetración de smartphones entre la población española alcanzó el 63\%, la tasa más alta de Europa (la media 
es de 55\%), un 15\% más que el año anterior. También según el estudio, más de un 10\% de los usuarios de smartphones accede diariamente a sitios de noticias, algo que se manifiesta en muchas de las respuestas analizadas y desvela la necesidad de muchos usuarios de acudir varias veces al día para conocer información actualizada.

Esta característica es precisamente una de las diferencias entre sendos formatos a las que más referencias hacen los encuestados en la segunda pregunta, junto a la interactividad, la inmediatez o la presencia de recursos visuales, todas ellas características evidentes del entorno digital. No obstante, cabe destacar que son varios los encuestados que creen que, en el fondo, los contenidos son similares tanto en la Red como en el papel. Esta opinión se basa en los volcados de información de una a otra edición que llevan a cabo algunas cabeceras, por ejemplo las pertenecientes al grupo editorial Joly, cuyo resultado es una información similar en ambos casos. A pesar de ello, la mayoría sigue usando los diarios digitales, en muchos casos, y según los datos recabados, por la gratuidad de sus contenidos y la posibilidad de comparar entre periódicos.

Esto ha posibilitado un incremento de la pluralidad informativa de los usuarios, que ya no sólo consultan un sólo diario, algo habitual con la prensa tradicional, sino que acuden a varios con el propósito de confrontar los distintos enfoques. Al menos eso es lo que se deriva de las respuestas de muchos de los encuestados, quienes admiten desconfiar de todas las cabeceras, lo cual corrobora a su vez la falta de credibilidad de los medios de comunicación entre la sociedad. A pesar de ello, cabe reseñar que la formación del alumnado encuestado puede realzar esta opinión, al conocer la profesión y la estructura empresarial de los medios desde más cerca. En un porcentaje menor, los alumnos y alumnas reconocen consultar de forma habitual un sólo medio, de acuerdo a una tendencia a la fidelización, ya sea por la costumbre o por el apego a la línea editorial, que se encuentra no obstante mucho más difuminada que en la prensa tradicional.

Una de las herramientas tecnológicas que ha incidido en esta dimensión son las redes sociales, en la medida en que facilitan el acceso desde una interfaz única que es administrada según las preferencias del usuario. La amplia mayoría del alumnado se reconoce consumidor asiduo de estas redes, aunque una parte de ellos advierte de que nos las utiliza con afán de obtener información. Entre sus razones, destaca la visión crítica mantenida respecto a los contenidos que se difunden por estos canales, caracterizados por cierta provisionalidad. El resto de usuarios que sí consumen información ensalzan las redes sociales como el medio de comunicación del siglo XXI, al ofrecer la posibilidad, de forma gratuita, de conocer los intereses de la sociedad y las últimas tendencias, imponiéndose cada uno su propia agenda informativa.

Cuando hablamos de compartir contenidos a través de estas redes, la discusión se amplía. Si bien la mayoría reconoce hacerlo, una parte importante de este grupo no comparte asiduamente, sino tan solo en momentos puntuales. Otro porcentaje relevante afirma no compartir contenidos nunca o casi nunca. No obstante, el objetivo general entre los que sí lo hacen es generar debate, extender la conversación entre su entorno, propiciar la denominada serendipia social, es decir, la recomendación de noticias aleatorias. De hecho, según un informe del Pew Internet \& American Life Project (2010) titulado Understanding the Participatory News Consumer, se destacaba que el $75 \%$ de los usuarios encuestados se informaba a través de enlaces compartidos 
por sus amigos y seguidores. De este modo, la gente usa las redes sociales para filtrar, evaluar y reaccionar ante las noticias (Purcell, 2010).

Pero esta participación también tiene consecuencias. Según el alumnado, este entorno participativo incide de forma sustancial en el desarrollo del debate democrático de la sociedad, pero desde un punto de vista profesional, deriva en problemas que afectan directamente a la identidad del periodista. En esta pregunta se desvela de forma clara la ambivalencia del alumnado frente al periodismo o participación ciudadana, en la medida en que, actualmente, ellos se encuentran insertos en una de las esferas y están próximos a pasar a otra profesional, donde la confusión de discursos y contenidos, periodísticos o no, les puede repercutir. Así pues, se advierte una polarización de los encuestados entre los que están a favor de esta participación y aquellos que no, situándose en medio un porcentaje importante que agrega diferentes matices a su respuesta y se sitúan en terreno de nadie.

No obstante, en la siguiente pregunta sí que colocan el intrusismo entre los dilemas éticos más importantes del entorno digital, sólo por detrás de la inmediatez. También cabe destacar la importancia dada a la necesidad de filtros u organismos que velen por el cumplimiento ético del periodismo, una posición que coincide con la investigación de Salvador Alsius (2010) entre periodistas catalanes. En torno a la mitad de estos defendían la necesidad de adoptar medidas coercitivas para que los códigos éticos, que consideraban poco o nada eficaces, sean llevados a la práctica. Es igualmente curioso hacer notar la escasa atención dada por los encuestado a la precariedad laboral, probablemente el principal problema actual de la profesión, con unos índices de desempleo desbocados.

A tenor de los resultados obtenidos, se puede apreciar un cambio evidente en los patrones de consumo de los jóvenes universitarios. La información digital y las redes sociales han sustituido paulatinamente a los medios analógicos y han cambiado la forma de relacionarse con los contenidos. Los usuarios acceden a una información más plural desde distintos dispositivos y a partir de un sistema de recomendación social semicontrolado por el propio usuario. Por otra parte, los futuros periodistas se debaten entre las bondades de la participación abierta de la ciudadanía en el entorno digital y los efectos perniciosos que esta puede desatar en la consideración de la profesión, igualmente puesta en cuestión por el exceso de sensacionalismo, la falta de veracidad o la inmediatez.

\section{Referencias bibliográficas}

ALSIUS, Salvador (Ed., 2010): "The Ethical values of journalists. Field research among the media professionals in Catalonia". Quaderns del CAC, núm.36, pp. 137-138.

BOWMAN, Shayne and WILLIS, Chris (2003): We Media: How Audiences are Shaping the Future of News and Information. The Media Center at the American Press Institute. En: http://www.hypergene.net/wemedia/download/we_media.pdf

BRUNS, Axel (2005): Gatewatching: Collaborative Online News Production. New York, Peter Lang. 
BRUNS, Axel (2011): "Oblique strategies for ambient journalism". Media Culture Journal, vol. 13, num. 2, en: http://www.journal.media-culture.org.au/index.php $/$ mcjournal/article/viewArticle/230

CASTELLS, Manuel (2009): Comunicación y Poder. Madrid, Alianza Editorial.

CIS (2013): Barómetro de Febrero. Estudio 2.978. Febrero 2013. En: http://datos.cis.es/pdf/Es2978mar_A.pdf

COMSCORE (2012): España muestra el mayor crecimiento de consumo de noticias desde smartphones de Europa. 22/3/2012. En: http://www.comscore.com/esl/Insights/Press_Releases/2012/3/Number_of_European_Smartphone_Users_Accessing_News_Surges_74_Percent_Over_Past_Year

DOMINGO, David and QUANDT, Thomas et al. (2008): "Participatory Journalism practices in the media and beyond". Journalism Practice, vol. 2, num. 3, pp. 326342.

DEUZE, Mark (2008): “Understanding journalism as network: How it changes, and how it remains the same". Westminster Papers in Communication and Culture, vol. 5, num. 2, pp. 4-23.

HARRISON, Jackie (2009): "User-generated content and gatekeeping at the BBC hub". Journalism studies, vol. 11, num. 2, pp. 243-256.

HERMIDA, Alfred (2010): "From TV to Twitter: How ambient news became ambient journalism". Media-Culture Journal, vol. 13, num. 2: http://papers.ssrn.com/sol3/papers.cfm?abstract_id=1732603

MESSNER, Marcus; LINKE, Maureen; \& ESFORD, Asriel (2011): "Shoveling tweets: An analysis of the microblogging engagement of traditional news organizations". Paper presented at International Symposium on Online Journalism. Austin, Texas, April 1-2.

OLMSTEAD, Kenneth; MITCHELL, Amy; \& ROSENSTIEL, Tom (2011): "Navigating news online", Pew Research Center's Project for excellence in Journalism. En: http://www.journalism.org/2011/05/09/navigating-news-online/

PURCELL, Kristen; RAINIE, Lee; MITCHELL, Amy; ROSENSTIEL, Tom; and OLMSTEAD, Kenny (2010): "Understanding the Participatory News Consumer" Pew Internet \& American Life Project: http://www.pewinternet.org/2010/03/01/understanding-the-participatory-news-consumer/

ROSEN, Jay (2006): "The people formerly known as the audience". The Huffington Post, [30-6-2006]. En: http://www.huffingtonpost.com/jay-rosen/the-people-formerly-known_1_b_24113.html

SINGER, Jane; HERMIDA, Alfred \& et al. (2011): Participatory Journalism: Guarding Open Gates at Online Newspapers. New York, Wiley-Blackwell.

TOFFLER, Alvin (1971): Future Shock. London, Pan. 